\title{
sciendo
}

\section{Heart Rate Variability is Correlated with Perceived Physical Fitness in Elite Soccer Players}

\author{
by \\ Guillaume Ravé1, Hassane Zouhal', Daniel Boullosa3,4, Patricia K. Doyle-Baker5, \\ Ayoub Saeidi ${ }^{6}$, Abderraouf Ben Abderrahman', Jacques-Olivier Fortrat ${ }^{1}$
}

Heart Rate Variability (HRV) has been typically used to monitor athletes' physical fitness readiness. The supine position maximizes parasympathetic tone, which is important for monitoring in continuous aerobic sports, however, this is not the case of team sports that rely on anaerobic intermittent bouts, thus increasing sympathetic activation and vagal withdrawal. We hypothesized that HRV during sympathetic activation and vagal withdrawal would be a useful marker to evaluate perceived physical fitness in team sports. HRV was measured in both supine and standing positions during the mornings of 4 match days in 14 professional players. The supine Root Mean Square of the Successive Differences (RMSSD), as well as spectral analysis indices were recorded. Perceived physical fitness was assessed after each match by means of a visual analogue scale (VAS). Supine RMSSD was moderately correlated with perceived physical fitness $(r h o=0.416)$, however, larger correlations were observed for supine and standing spectral indices (rho > 0.5). Correlation between RMSSD and Total Power was very large, thus questioning the usual interpretation of RMSSD (rho > 0.7). Standing Spectral HRV analyses may be a useful method for evaluating perceived physical fitness in the context of team sports. RMSSD may reflect the overall variability of HR and not only the parasympathetic influence, as observed in the current study.

Key words: autonomic nervous system, soccer, training, intermittent sports, orthostatic test.

\section{Introduction}

A professional soccer season has a duration of nine to eleven months involving 50-80 matches per year that are played once or twice a week (Mohr et al., 2015). Maintaining a high level of physical fitness and avoiding fatigue and injuries is critical for players' ongoing performances. Identifying and predicting decreases in physical performance would be helpful for coaches as training loads could be modified accordingly. It is therefore essential to have reliable and valid indicators of players' adaptation to training and competitive loads. There are a number of scientifically validated tools currently available for this purpose. Neuromuscular assessments (e.g. counter movement jump, tensiomyography), physical data (e.g. distances travelled, number of accelerations), subjective data (e.g., perceived physical fitness, rating of perceived exertion) and recovery stress questionnaires (e.g. REST-Q), among others, are frequently used to monitor players' adaptation to training and game loads (Halson, 2014). In this context, a simple, time-

1 - CNRS 6214 Inserm 1083 Faculté de Médecine 49045 Angers, France.

2 - Univ Rennes, M2S (Laboratoire Mouvement, Sport, Santé) - EA 1274, F-35000 Rennes, France.

3 - Faculty of Health Sciences, University of Brasilia, Brasilia, Brazil.

4 - Sport and Exercise Science, James Cook University, Townsville, Australia.

5 - Human Performance Lab, Faculty of Kinesiology, University of Calgary, Calgary, Alberta, Canada.

6 - Department of Biological Sciences in Sport, Faculty of Sports Sciences and Health, Shahid Beheshti University, Tehran, Iran.

7 - Higher Institute of Sport and Physical Education, Ksar Said, Tunisia. 
efficient and valid tool for monitoring players' physical fitness would be an interesting addition to coaching from the perspective of game day player selection and manipulating training loads.

Heart rate variability (HRV) represents a simple method used to assess the autonomic nervous system (ANS) activity on the heart (Task Force of the European Society of Cardiology and The North American Society of Pacing and Electrophysiology, 1996). It provides sympathetic and parasympathetic influences through calculations based on heart rate (HR) recordings (Task Force of the European Society of Cardiology and The North American Society of Pacing and Electrophysiology, 1996). HRV has been widely used in training monitoring of athletes (Bellenger et al., 2016). Aerobic training mainly alters parasympathetic indices derived from time or from spectral analyses and these indices may contribute to predict endurance performance (Buchheit et al., 2010). These indices are usually assessed on supine rest since this position is known to maximize parasympathetic nervous system (PNS) activity (Abad et al., 2017; Silva et al., 2017; Young and Leicht, 2011). Among these parasympathetic indices, the Roots Mean Square of the Successive Differences (RMSSD) has been widely used given its robustness for aerobic training monitoring (Buchheit, 2014). However, previous studies in team sports showed contradictory results with regard to RMSSD (Flatt et al., 2016, 2017; Nakamura et al., 2015; Ravé and Fortrat, 2016). For instance, its logarithmic version (Ln RMSSD) after submaximal efforts seems useless for identifying players' adaptation to training loads (Thorpe et al., 2016).

These contradictory results could result from the fact that performance in team sports depends on various physical fitness components (e.g. speed, agility) apart from aerobic capacity, with match activities including random alternations between resting and active bouts that require both aerobic and anaerobic pathways. Such is the case of soccer, which could be considered as an "intermittent sport" (Bangsbo, 1994). Therefore, monitoring vagal modulations would be insufficient in soccer given that performance depends more on abilities than aerobic capacity (Ravé and Fortrat, 2016).

The influence of sympathetic nervous system (SNS) activity, in combination with PNS activity, is usually studied with HRV during different manoeuvres performed in order to activate the organism during a physiological challenge. These manoeuvres could activate the sympathetic nervous system through a central pathway (e.g. mental stress), a metabolic pathway (e.g. isometric exercise), or an integrative physiological response as occurs during adaptation to the standing position from the supine position (i.e. orthostatic test) (Olufsen et al., 2008). Previously, several studies have showed that the use of an orthostatic test during training monitoring improves the characterisation of acute and chronic training adaptations (Schmitt et al., 2015). For instance, recording during standing is needed to show the long-term HRV decrease after an interval training session (Mourot et al., 2004). We have also recently demonstrated that a standing position is needed to follow up the chronic training adaptations in professional soccer players (Ravé and Fortrat, 2016). Furthermore, Schmitt et al. (2015) characterised different types of fatigue when analysing HRV in both supine and standing positions. Therefore, contrary to aerobic sports, in which it seems that vagal modulations in the supine position could be sufficient to monitor training adaptations, it would be suggested that training monitoring throughout HRV with an orthostatic test could be more convenient when looking for complex adaptations as in soccer.

There is a need to assess perceived physical fitness of athletes during training and match days for better planning the training loads and squad selections, respectively. Physical performance can be measured with objective measures such as distance covered or time completed at different intensities, which are objectives measures of physical fitness, yet in team sports, performance depends not only on physical fitness, but also on situational factors like tactics, scoring and refereeing (Lago-Penas, 2012). In addition, it remains to be clarified whether the parasympathetic and sympathetic adaptations to training are linked to perceived physical fitness. Therefore, we hypothesized that HRV measured both in the supine and in the standing position would be related to the perceived physical fitness in professional soccer players, with stronger correlations observed in standing $\mathrm{HRV}$ as it is influenced by both vagal and sympathetic modulations. 


\section{Methods}

\section{Participants}

Fourteen soccer players from the French soccer league 'Ligue 2' (age 27.9 \pm 4.3 years, body height $1.79 \pm 0.04 \mathrm{~m}$, body mass $75.2 \pm 6.8 \mathrm{~kg}$, $\mathrm{VO}_{2 \max }$ of $58 \pm 4 \mathrm{ml} \cdot \mathrm{kg}^{-1} \cdot \mathrm{min}^{-1}$ ) agreed to participate in this study. Ethical approval was obtained from the ethical committee and the scientific council of the University of Angers (France) and informed consent was provided by each participant after detailed explanation of the study design and purposes.

\section{Orthostatic test}

Four matches were selected over twelve days during the in-season competitive period (Figure 1). Players completed a standardized orthostatic test prior to each match, at 8:30 am, 20 min before breakfast. Each test involved resting for 10 minutes in the supine position followed by 7 minutes of standing, without speaking or moving. A telemetric system (PolarTeamSystem $2^{\circledR}$, Polar Electro Oy, Kempele, Finland) was used to record R-R intervals during the test.

\section{Visual Analogue Scale (VAS)}

The VAS is a widely used tool in sport and clinical settings (Bijur et al., 2001; Crowcroft et al., 2015; Le Meur et al., 2013; Zurutuza et al., 2017). The VAS used in the current study was a metershaped scale of $10 \mathrm{~cm}$ with a triangle drawn on the other side. The vertex and the base indicated the minimum and the maximum scores, respectively. During evaluations, the player positioned a cursor along the triangle according to his perceived physical fitness. Thereafter, the researcher read the cursor position in centimeters on the other side of the VAS. The cursor position in centimeters was considered as the score (Scott and Huskinsson, 1976). Immediately after each match, players reported their level of perceived physical fitness regarding the match using this procedure.

\section{Heart Rate Variability analyses}

HRV time and frequency analyses were carried out to obtain parasympathetic and sympathetic influences on HR using custom designed software (aHRV v11.0.4, Nevrokard Kiauta $^{\circledR}$, Slovenia). Spectral power density was determined by means of frequency analysis of 512 RR-interval series after re-sampling at $2 \mathrm{~Hz}$ in both the supine and the standing position from RR recordings. The first two minutes of each position were deleted to avoid HR drifts related to cardiovascular adaptations (Wieling and Wesseling, 1993). The breathing rate was not controlled nor recorded. Artefacts and ectopic beats were filtered manually. The time domain HRV index selected for analyses was RMSSD. Spectral power $\left(\mathrm{ms}^{2} / \mathrm{Hz}\right)$ of Very Low (VLF $\leq 0.04$ $\mathrm{Hz})$, Low $(0.04 \mathrm{~Hz} \leq \mathrm{LF} \leq 0.15 \mathrm{~Hz})$ and High $(0.15$ $\mathrm{Hz} \leq \mathrm{HF} \leq 0.40 \mathrm{~Hz}$ ) Frequencies, as well as the Total power (or overall HRV, TP $\leq 0.4 \mathrm{~Hz}$ ), normalised LF and HF power (LFnu and HFnu ), and the LF/HF ratio, were also analysed as recommended by the Task Force (1996).

\section{Statistical analyses}

Data were expressed as mean values and included standard error of the mean (SEM; \pm ). The Spearman rank test (rho) was used in this study because perceived physical fitness was assessed by means of a narrow range scale (i.e. $0-10$ ), and to assess the relationships between perceived physical fitness and HR, RMSSD, TP, VLF, HF, LF, HFnu, LFnu, and LF/HF ratio (SPSS 20.0; SPSS Inc, Chicago). The magnitude of the correlations was defined according to the Hopkins's scale (Hopkins et al., 2009): $\mathrm{r}<0.1$, trivial; 0.1-0.3, small; $>0.3-0.5$, moderate; $>0.5-0.7$, large; $>0.7-0.9$, very large; $>0.9$, nearly perfect; and 1, perfect. Statistical significance was set at $p<0.05$.

\section{Results}

The quality of HR recordings was good (Figure 1 ) and in the normal range (Table 1). A moderate correlation was observed between RMSSD in the supine position and perceived physical fitness (Figure 2). Only one frequency analysis index (i.e. LF) showed a large correlation with perceived physical fitness in the supine position (Table 1) while two of them (i.e. LF and TP) showed a large correlation in the standing position (Table 1 and Figure 2). In the supine position, the correlation between parasympathetic indices, RMSSD and HFnu, was small and non-significant (Table 2). In contrast, correlations between RMSSD and HF, and between RMSSD and TP, were very large (Table 2).

\section{Discussion}

Our study goal was to investigate the relationship between HRV indices and perceived physical fitness. Spectral analysis indices showed the strongest correlations with perceived physical 
fitness when compared to supine RMSSD, especially when HRV was assessed in the standing position. Soccer players in this study were able to perceive a greater physical fitness when their overall HRV (i.e. TP) was high in both supine and standing positions, demonstrating appropriate readiness for competition.

Table 1

Correlation between Physical fitness and Heart Rate Variability.

\begin{tabular}{|c|c|c|c|c|c|}
\hline & Indices & mean $\pm \mathrm{sd}$ & Spearman $\mathrm{r}$ & magnitude & $p$ \\
\hline & Physical Fitness (a.u) & $7.8 \pm 0.2$ & & & \\
\hline \multirow[t]{9}{*}{ Supine } & HR (bpm) & $57.1 \pm 1.1$ & -0.074 & - & ns \\
\hline & RMSSD (ms) & $53.2 \pm 4.4$ & 0.416 & moderate & $* *$ \\
\hline & $\mathrm{T}\left(\mathrm{ms}^{2}\right)$ & $3668.8 \pm 521.3$ & 0.455 & moderate & $* *$ \\
\hline & $\operatorname{VLF}\left(\mathrm{ms}^{2}\right)$ & $1213.6 \pm 182.7$ & 0.302 & - & ns \\
\hline & $\mathrm{LF}\left(\mathrm{ms}^{2}\right)$ & $1440.6 \pm 194.7$ & 0.524 & large & $* * *$ \\
\hline & LFnu (n.u) & $58.7 \pm 2.2$ & 0.390 & moderate & $* *$ \\
\hline & $\mathrm{HF}\left(\mathrm{ms}^{2}\right)$ & $1014.5 \pm 200.7$ & 0.309 & - & ns \\
\hline & HFnu (n.u) & $41.3 \pm 2.2$ & -0.396 & moderate & $* *$ \\
\hline & $\mathrm{LF} / \mathrm{HF}$ & $1.8 \pm 0.2$ & 0.391 & moderate & $* *$ \\
\hline
\end{tabular}

Standing

\begin{tabular}{|c|c|c|c|c|}
\hline HR (bpm) & $71.2 \pm 1.5$ & -0.090 & - & ns \\
\hline RMSSD (ms) & $35.1 \pm 17.8$ & 0,478 & moderate & $* *$ \\
\hline $\mathrm{T}\left(\mathrm{ms}^{2}\right)$ & $5792.3 \pm 608.0$ & 0.558 & large & $* * *$ \\
\hline $\operatorname{VLF}\left(\mathrm{ms}^{2}\right)$ & $2776.5 \pm 371.5$ & 0.477 & moderate & $* *$ \\
\hline $\mathrm{LF}\left(\mathrm{ms}^{2}\right)$ & $2542.7 \pm 259.0$ & 0.552 & large & $* * *$ \\
\hline LFnu (n.u) & $85.7 \pm 1.2$ & -0.064 & - & ns \\
\hline $\mathrm{HF}\left(\mathrm{ms}^{2}\right)$ & $473.0 \pm 76.8$ & 0.442 & moderate & $* *$ \\
\hline HFnu (n.u) & $14.3 \pm 1.2$ & 0.064 & - & ns \\
\hline LF/HF & $9.2 \pm 1.1$ & -0.065 & - & ns \\
\hline
\end{tabular}

Heart rate $(H R)$ and Heart Rate Variability (HRV) indices (mean \pm SEM) in the supine and standing positions before a soccer match. The correlation between perceived physical fitness and the HRV index is mentioned as well as the magnitude of the correlation. RMSSD: Root Mean Square of the Successive Differences; TP: Total power; VLF: Very Low Frequency; LF: Low Frequency; HF: High Frequency; nu: normalized units. ns: non-significant, ${ }^{* *} p<0.01,{ }^{* * *} p<0.001$ 
Table 2

Correlation between RMSSD and spectral parasympathetic indicators.

\begin{tabular}{llllll}
\hline \multirow{2}{*}{ Indicators } & $\begin{array}{l}\text { Spearman } \\
\mathrm{r}\end{array}$ & magnitude & $p$ & slope \\
\hline Supine & & & & & \\
& & & & & \\
& TP $\left(\mathrm{ms}^{2}\right)$ & 0.861 & Very large & $* * *$ & +110.53 \\
HF $\left(\mathrm{ms}^{2}\right)$ & 0.938 & Very large & $* * *$ & +40.71 \\
HFnu (n.u) & 0.024 & - & $\mathrm{ns}$ & +0.06 \\
\hline
\end{tabular}

Correlation between several Heart Rate Variability indices derived from time and frequency analyses that are influenced by the parasympathetic nervous system as well as the magnitude of the correlation. Mean and SEM are mentioned in the Table 1. T: Total power; RMSSD: Root Mean Square of the Successive Differences; HF: High Frequency power; HFnu: normalized power of High Frequency domain (n.u.). ns: non-significant, ${ }^{* *} p<0.01,{ }^{* * *} p<0.001$

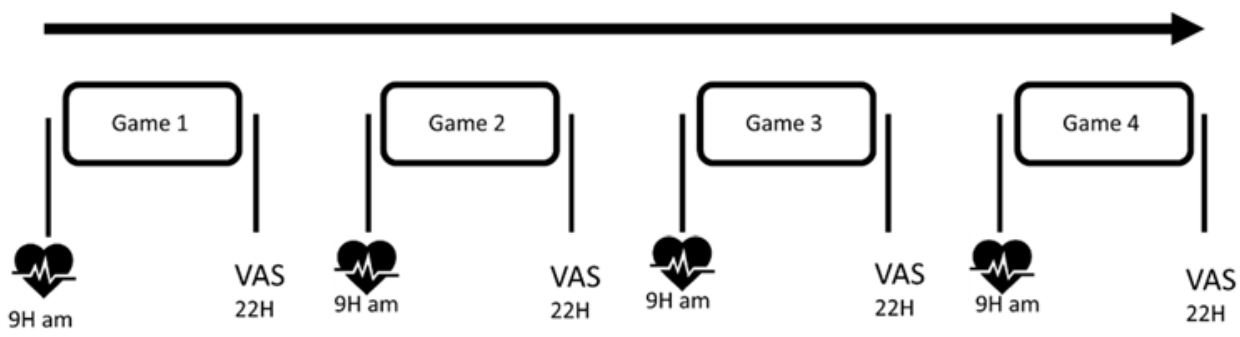

Heart rate recording; VAS : Visual analogic scale

Figure 1

Experimental design

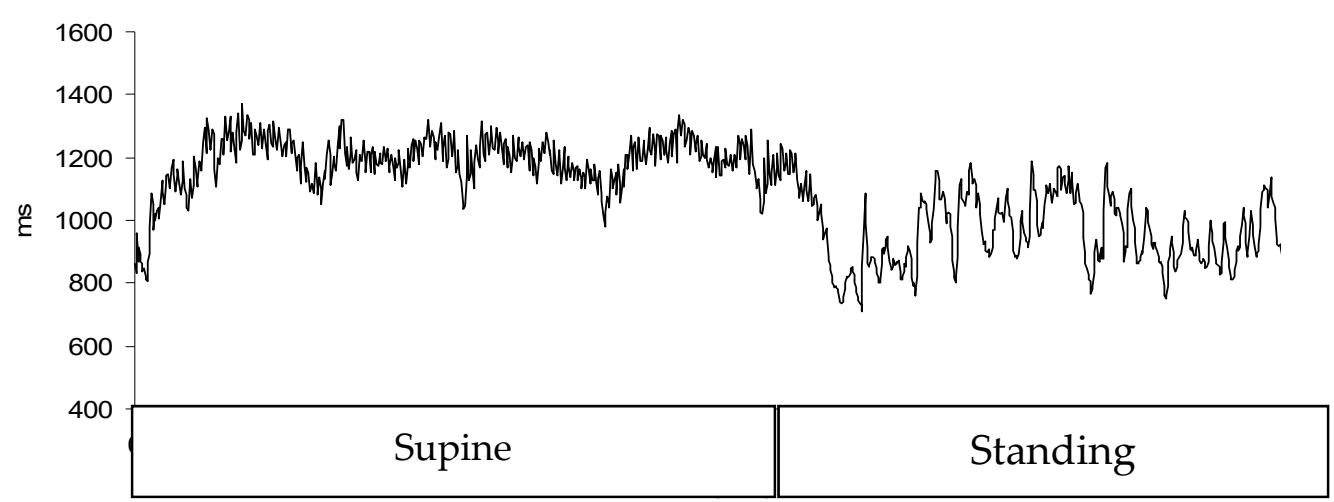

Figure 2

Heart rate recording. Heart rate recording of a player during a stand test before a competition match. The stand test included a 10 min supine period followed by a $7 \mathrm{~min}$ standing period. 


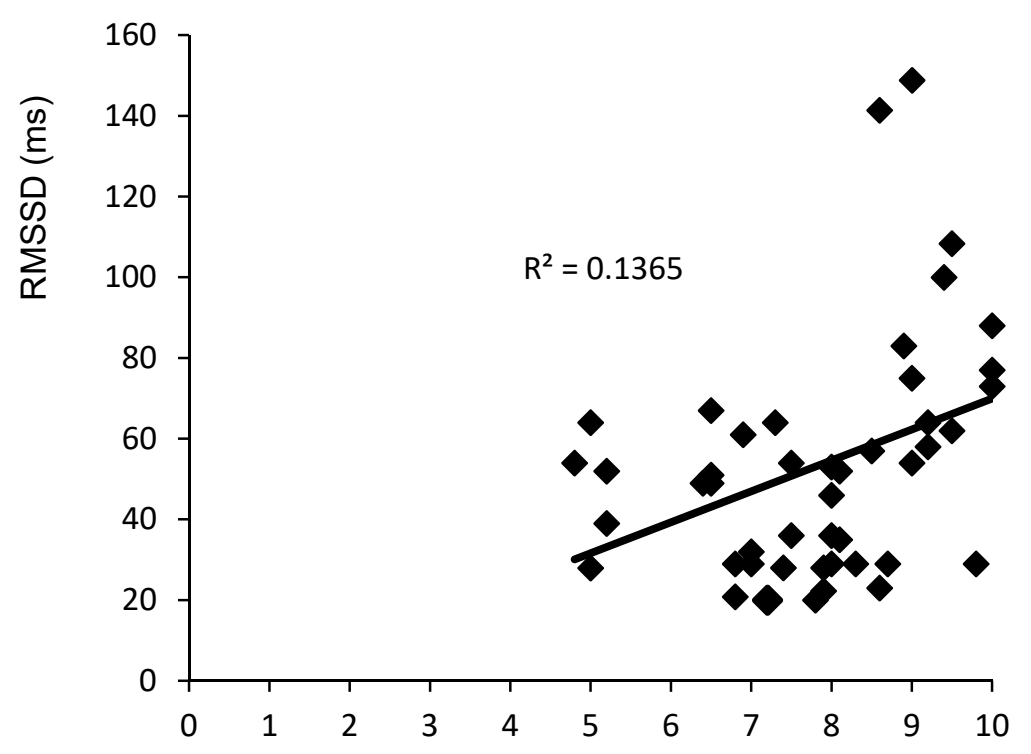

Physical Fitness Perception

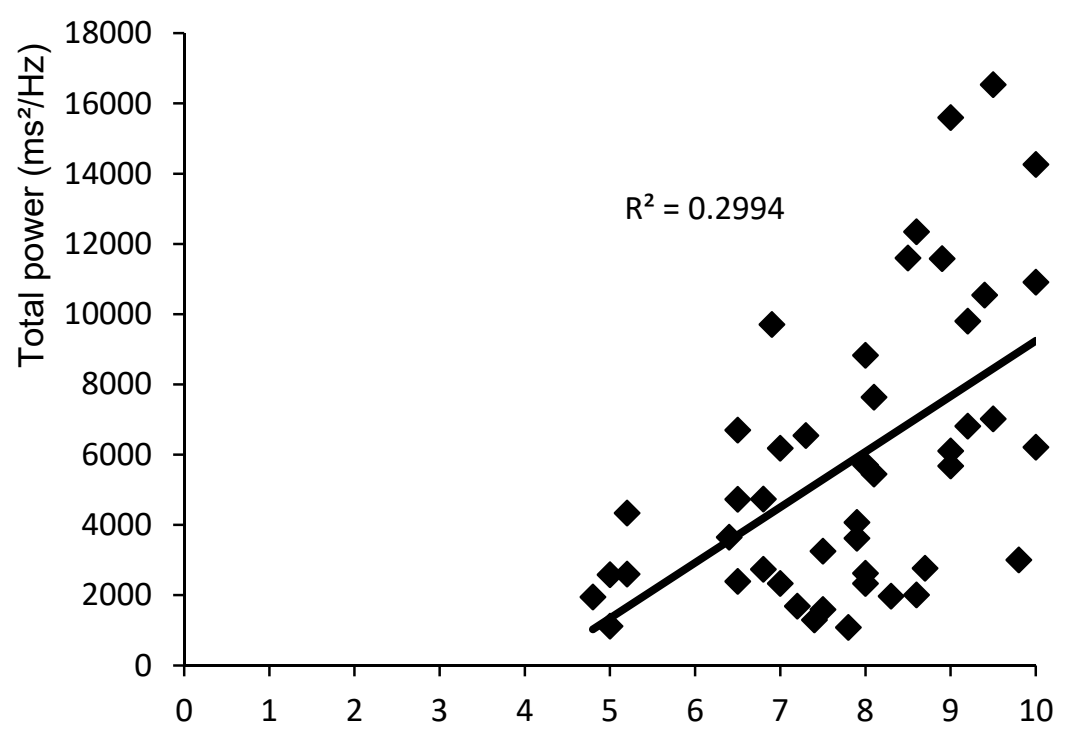

Physical Fitness Perception

Figure 3

Correlation between Heart Rate Variability and perceived physical fitness. Panel A: correlation between Root Mean Square of the Successive Differences (RMSSD, ms) in the supine position and physical fitness (arbitrary units, A.U.). Panel B: correlation between Total power of Heart Rate Variability in the standing position $\left(\mathrm{ms}^{2} / \mathrm{Hz}\right)$ and perceived physical fitness (A.U.). 
Players' physical activity during official matches varies from match to match (Gregson et al., 2010) because of a number of situational factors (Lago-Penas, 2012). Therefore, physical fitness may not be determined by means of tools that quantify physical activity during a single match because of this high variability. This means that, for appropriate comparisons, a number of matches would be necessary for achieving an acceptable signal-to-noise ratio. Thus, we decided to directly quantify physical fitness as subjectively perceived by players to overcome this limitation. Perceived physical fitness was quantified in our study by means of a VAS. The VAS is widely and daily used in medicine mainly to assess pain (Hawker et al., 2011). The VAS is also used in sport sciences. For instance, Grant et al. (1999) showed that a VAS is reliable and sensitive to assess physical fitness changes during submaximal exercise. In another study, VAS was used to show that perceived physical fitness and both aerobic and strength fitness components were significantly correlated (Hauser and Holley, 2013). Furthermore, the VAS has been also used in the context of soccer to assess muscular pain, fatigue, sleep quality, and mood (Shearer et al., 2017). Therefore, perceived physical fitness evaluated via the VAS could be considered an appropriate tool for our purposes. Meanwhile, further studies should be conducted to elaborate on the construct validity and reliability of this tool.

Training monitoring in endurance sports needs to focus on parasympathetic indices, mainly RMSSD (Edmonds et al., 2013). However, in team sports, training monitoring should also take into account indices with some sympathetic components (i.e. LF and LFnu), which were correlated with perceived physical fitness in the current study. Moreover, the parasympathetic index HFnu was inversely correlated with perceived physical fitness. Furthermore, the standing position improved correlations between HRV indices and perceived physical fitness (Table 1). Standing sympathetic activation was demonstrated with increases in HR, LF, LFnu, and LF/HF (20, 43, 31, and 80\%, respectively). Our results are in accordance with previous studies showing that standing HRV better reflects training loads of soccer players while usual supine HRV indices remain unchanged (Ravé and
Fortrat, 2016).

RMSSD is the HRV index most used to monitor aerobic training (Plews et al., 2013). This index is a robust parasympathetic modulation variable (Task Force of the European Society of Cardiology and The North American Society of Pacing and Electrophysiology, 1996). Our study showed that RMSSD was correlated with perceived physical fitness in a team sport and confirmed that RMSSD could be a useful marker of adaptation in the context of team sports. HF is under the dependence of parasympathetic influences and is also known to be correlated with RMSSD (Task Force of the European Society of Cardiology and The North American Society of Pacing and Electrophysiology, 1996). The current study showed, however, an inverse correlation between perceived physical fitness and HFnu. Unexpectedly, RMSSD and HFnu, two recognized parasympathetic modulation variables, were not correlated to each other. Moreover, RMSSD was strongly correlated with TP, an index of overall HRV, in the supine position. Unlike HFnu, RMSSD does not benefit from normalisation according to overall HRV influences (Task Force of the European Society of Cardiology and The North American Society of Pacing and Electrophysiology, 1996). Therefore, the large correlation between RMSSD and overall HRV may suggest that RMSSD better reflects the overall HRV and not the parasympathetic influences in team sports such as soccer. Further studies using appropriate stimuli and selective autonomic blockades would confirm the validity of the current results.

We sought to identify potential and useful markers of training adaptation in team sports using HRV indices in both supine and standing positions. Supine RMSSD was only moderately correlated with perceived physical fitness in soccer players, however, other HRV indices as TP in a standing position or LF in supine and standing positions showed stronger correlations and therefore are likely to be better markers than RMSSD. Unfortunately, no single index demonstrated a strong correlation with perceived physical fitness. This was not unexpected since HRV is not influenced only by physical fitness levels. HRV recordings may have been influenced by the players' anxiety on the morning of the competition. Anxiety alters the 
sympathovagal balance (Mateo et al., 2012). For instance, Edmonds et al. (2013) previously observed a decrease in RMSSD, HF and HFnu, and an increase in LF on the day of a rugby match. In addition, HRV indices can only evaluate the autonomic nervous system activity while perceived physical fitness may be also under the dependence of other physiological systems like the neuromuscular system. The latter is very important in soccer since accelerations, sprints, brakings, and changes of direction cause neuromuscular fatigue, with players' physical performances negatively correlated to muscular training loads (Arcos et al., 2015). Moreover, Buchheit et al. (2012) previously observed that parasympathetic HRV indices were linked to cardiorespiratory testing, but not to neuromuscular testing. Further studies should take into account both cardiovascular and neuromuscular system evaluations in order to identify a reliable marker of perceived physical fitness for training monitoring.

In conclusion, HRV has a great potential as a useful maker of physical fitness readiness in team sports. Further studies are needed to determine whether different combinations of HRV indices could efficiently predict the influence of different physical fitness components on specific performance, with more studies also needed to determine whether a combination of HRV and neuromuscular indices might improve physical fitness readiness evaluation.

\section{Practical implications}

$-H R V$ in the morning may represent a valid marker of physical fitness readiness in soccer players.

-HRV in the standing position may be a better monitoring tool than supine HRV in sports taxing simultaneously different physical capacities.

\section{Acknowledgements}

The authors thanks all the players and the staff for their partcipation.

Founding: JOF benefits from the support of the Centre National d'Études Spatiales (National Centre for Spatial Studies) (CNES, grant \# 2014/4800000763).

\section{References}

Abad C, Kobal R, Kitamura K, Gil S, Pereira L, Loturco I, Nakamura F. Heart rate variability in elite sprinters: effects of gender and body position. Clin Physiol Funct Imaging, 2017; 37(4): 442-447

Akselrod S, Gordon D, Ubel FA, Shannon DC, Berger AC, Cohen RJ. Power spectrum analysis of heart rate fluctuation: a quantitative probe of beat-to-beat cardiovascular control. Science, 1981; 213(4504): 220222

Arcos AL, Martínez-Santos R, Yanci J, Mendiguchia J, Méndez-Villanueva A. Negative associations between perceived training load, volume and changes in physical fitness in professional soccer players. J Sports Sci Med, 2015; 14(2): 394-401

Bangsbo J. The physiology of soccer with special reference to intense intermittent exercise.Acta Physiologica Scandinavia, 1994; 151(619), 1-156

Bellenger CR, Fuller JT, Thomson RL, Davison K, Robertson EY, Buckley JD. Monitoring athletic training status through autonomic heart rate regulation: A systematic review and meta-analysis. Sports Med, 2016; 46(10): 1461-1486

Bijur PE, Silver W, Gallagher EJ. Reliability of the visual analog scale for measurement of acute pain. Acad Emerg Med, 2001; 8(12): 1153-1157

Buchheit M, Chivot A, Parouty J, Mercier D, Al Haddad H, Laursen PB, Ahmaidi S. Monitoring endurance running performance using cardiac parasympathetic function. Eur J Appl Physiol, 2010; 108(6): 11531167

Buchheit M, Simpson MB, Al Haddad H, Bourdon PC, Mendez-Villanueva A. Monitoring changes in physical performance with heart rate measures in young soccer players. Eur J Appl Physiol, 2012; 112(2): 711-723 
Buchheit M. Monitoring training status with HR measures: do all roads lead to Rome? Front Physiol, 2014; 5: 73

Crowcroft S, Duffield R, McCleave E, Slattery K, Wallace LK, Coutts AJ. Monitoring to assess changes in fitness and fatigue: The effects of training in heat and hypoxia. Scand J Med Sci Sports, 2015; 25(Suppl 1): 287-295.

Edmonds RC, Sinclair WH, Leicht AS. Effect of a training week on heart rate variability in elite youth rugby league players. Int J of Sport Med, 2013; 34(12): 1087-1092

Flatt AA, Esco MR, Nakamura FY, Plews DJ. Interpreting daily heart rate variability changes in collegiate female soccer players. J Sports Med Phys Fitness, 2016; 57(6): 907-915

Flatt AA, Esco MR, Nakamura FY. Individual Heart Rate Variability Responses to Preseason Training in High Level Female Soccer Players. J Strength Cond Res, 2017; 31(2): 531-538

Grant S, Aitchison T, Henderson E, Christie J, Zare S, Murray J, Dargie H. A Comparison of the Reproducibility and the Sensitivity to Change of Visual Analogue Scales, Borg Scales, and Likert Scales in Normal Subjects During Submaximal Exercise. Chest, 1999; 116(5): 1208-1217

Gregson W, Drust B, Atkinson G, Salvo VD. Match-to-match variability of high-speed activities in premier league soccer. Int J Sports Med, 2010; 31(4): 237- 42

Halson SL. Monitoring training load to understand fatigue in athletes. Sports Medicine, 2014; 44 Suppl 2:S 139-147

Hauser T, Aaron H. Self-Assessment Using a Validated Visual Analogue Scale Predicts Response to Submaximal Exercise Testing. Chest, 2013; 144(4)

Hawker A, Mian S, Kendzerska T, French M. Measures of adult pain: Visual Analog Scale for Pain (VAS Pain), Numeric Rating Scale for Pain(NRS Pain), McGill Pain Questionnaire (MPQ), Short-Form McGill Pain Questionnaire (SF-MPQ), Chronic Pain Grade Scale (CPGS), Short Form-36 Bodily Pain Scale (SF-36 BPS), and Measure of Intermittent and Constant Osteoarthritis Pain (ICOAP). Arthritis Care Res, 2011; 63: 240-252.

Hopkins WG, Marshall SW, Batterham AM, Hanin J. Progressive statistics for studies in sports medicine and exercise science. Med Sci Sports Exerc, 2009; 41(1): 3-13

Lago-Peñas C. The role of situational variables in analysing physical performance in soccer. J Hum Kinet, 2012; 35: 89-95

Le Meur Y, Pichon A, Schaal K, Schmitt L, Louis J, Gueneron J, Hausswirth C. Evidence of parasympathetic hyperactivity in functionally overreached athletes. Med Sci Sports Exerc, 2013; 45(11): 2061-2071

Mateo M, Blasco-Lafarga C, Martínez-Navarro I, Guzmán JF, Zabala M. Heart rate variability and precompetitive anxiety in BMX discipline. Eur J Appl Physiol, 2012; 112(1): 113-123

Mohr M, Draganidis D, Chatzinikolaou A, Barbero-Álvarez JC, Castagna C, Douroudos

I, Avloniti A, Margeli A, Papassotiriou I, Flouris AD, Jamurtas AZ, Krustrup P, Fatouros IG. Muscle damage, inflammatory, immune and performance responses to three football games in 1 week in competitive male players. Eur J Appl Physiol, 2015; 116(1): 179-193

Mourot L, Bouhaddi M, Tordi N, Rouillon JD, Regnard J. Short- and long-term effects of a single bout of exercise on heart rate variability: comparison between constant and interval training exercises. Eur J Appl Physiol, 2004; 92(4-5): 508-517

Nakamura FY, Flatt AA, Pereira LA, Ramirez-Campillo R, Loturco I, Esco M. Ultra-Short-Term Heart Rate Variability is Sensitive to Training Effects in Team Sports Players. J Sports Sci Med, 2015; 14(3): 602-605

Olufsen MS, Alston AV, Tran HT, Ottesen JT, Novak V. Modeling heart rate regulation part I: sit-to-stand versus head-up tilt. Cardiovasc Eng, 2008; 8: 73-87

Plews DJ, Laursen PB, Stanley J, Kilding AE, Buchheit M. Training adaptation and heart rate variability in elite endurance athletes: opening the door to effective monitoring. Sports Med, 2013; 43(3): 773-781

Ravé G, Fortrat JO. Heart Rate Variability in the standing position reflects training adaptation in professional soccer players. Eur J Appl Physiol, 2016; 116(8): 1575-1582

Schmitt L, Regnard J, Parmentier AL, Mauny F, Mourot L, Coulmy N, Millet GP. Typology of "Fatigue" by Heart Rate Variability Analysis in Elite Nordic-skiers. Int J Sports Med, 2015; 36(12): 999-1007 
Silva CC, Bertollo M, Reichert FF, Boullosa DA, Nakamura FY. C.C. Reliability of Heart Rate Variability in Children: Influence of Sex and Body Position During Data Collection. Pediatr Exerc Sci, 2017; 29(2): 228-236

Scott J, Huskisson E. Graphic representation of pain. Pain, 1976; 2(2): 175-184

Shearer DA, Sparkes W, Northeast J, Cunningham DJ, Cook CJ, Kilduff LP. Measuring recovery: An adapted Brief Assessment of Mood (BAM+) compared to biochemicaland power output alterations. J Sci Med Sport, 2017; 20(5): 512-517

Task Force of the European Society of Cardiology and The North American Society of Pacing and Electrophysiology. Heart rate variability Standards of measurement, physiological interpretation, and clinical use. Eur Heart J, 1996; 17(3): 354-381

Thorpe RT, Strudwick AJ, Buchheit M, Atkinson G, Drust B, Gregson W. The Tracking of Morning Fatigue Status Across In-Season Training Weeks in Elite Soccer Players. Int J Sports Physiol Perform, 2016; 11(7): 947-952 Wieling W, Wesseling KH. Importance of reflexes in the circulatory adjustments changes. In: Hainsworth R, Mark AL (eds) Cardiovascular reflex control in health and disease. Saunders, London, 35-63; 1993

Young FL, Leicht AS. Short-term stability of resting heart rate variability: influence of position and gender. Appl Physiol Nutr Metab, 2011; 36(2): 210-218

Zurutuza U, Castellano J, Echeazarra I, Casamichana D. Absolute and Relative Training Load and Its Relation to Fatigue in Football. Front Psychol, $2017 ; 6(8): 878$

\section{Corresponding author:}

\section{Prof. H. ZOUHAL (PhD)}

University of Rennes 2

M2S (Laboratoire Mouvement, Sport, Santé) - EA 1274,

F-35000 Rennes, France

E-mail: hassane.zouhal@univ-rennes2.fr 